\title{
ETHNICITÉ, NOURRITURE ET POLITIQUE L'EXEMPLE DES YASA DE LA CÔTE SUD DU CAMEROUN
}

\author{
Flavien TIOKOU NDONKO
}

BP 2271

Messa

Yaoundé, Cameroun

\section{SUMMARY}

ETHNICITY, FOOD, AND POLITICS: THE EXAMPLE OF THE YASA IN THE CAMEROONIAN SOUTH PROVINCE

In Cameroon, ethnicity is present in food as well as in anthroponymy. Neighbouring populations use it to auto-identify themselves and to indicate their spatial settlement. The 'leaves'eaters', the 'crabs'eaters' and the '"fruits de l'arbre a pain"-eaters' live together in peace on the Coast of the country by assuming their ethnicity. They even take over new political and administrative structures as guide mark in self presentation. Even the political power who officially tries to 'erase' ethnicity is not able to compose without it. Ethnicity has become a political strategy and is being mobilized or demobilized to balance or control the power.

KEY WORDS: Ethnicity, food, politics, space, stereotype 


\section{Introduction}

" "You Bapende... you kill dogs to eat them". "Well...you Bayanzi can't talk; you eat men". This remark caused an outburst of indignation... "It is all very well to eat your enemies when you have killed them in battle -is not that quite a natural thing to do? but no descent person would think of eating his friend. You Bapende think nothing of eating dogs, the greatest friend of man" , (Frank, 1964:74-5).

Cet exemple extrême, emprunté à Hilton-Simpson, qui parle de deux groupes ethniques voisins dans le Kasai (Zaïre actuel), l'un cynophage et l'autre anthropophage, montre à quel point l'animosité inter-ethnique prend souvent des formes d'accusation portant sur les habitudes alimentaires. Au Cameroun, les populations se servent aussi de la nourriture pour identifier et classer les groupes qui les entourent. Autrement dit, c'est par ce que les uns et les autres mangent, qu'on circonscrit l'appartenance à une ethnie ou à une partie du territoire national. Mais quand la composition d'une région éloignée du pays est ignorée, on se sert de la religion ou d'une langue pour étiqueter les groupes qui y habitent. Qu'il nous soit permis ici d'isoler les aspects socio-culturels de la nourriture de leur aspect physiologique afin de montrer quelques usages symboliques qu'on fait du 'manger'.

Les données sur lesquelles est basée cette réflexion ont été collectées entre 1987-90 dans les villages côtiers du Sud Cameroun. J'y effectuais alors une recherche comparative sur les modes d'occupation et de gestion de l'espace en vue d'explorer les stratégies traditionnelles d'évacuation des excréments et des autres déchets corporels. Cette étude sera présentée sous forme de dissertation doctorale au semestre d'hiver 92 à l'Université de Hambourg (Allemagne).

\section{Le Cameroun dans le plat}

Où qu'on se trouve au Cameroun l'évocation d'un aliment fait penser à une ethnie ou à l'une des dix provinces du pays, quand on ignore les groupes culturels, parfois très nombreux, qui les composent. Il n'y a pas très longtemps un musicien faisait danser les Camerounais au rythme de l'alimentation des principaux groupes ethniques: si tu aimes un Bassa prépare lui le mbongo-tsobi, sinon le mariage n'ira 
pas bien; si tu aimes un Bamiléké prépare lui le nkui, sinon... Ces évocations sont assez significatives, car dans les représentations des populations le mbongo-tsobi (sauce noire de poisson) correspond à l'ethnie bassa. N'empêche que les non-Bassa adorent ce plat 'traditionnel' qu'ils vont jusqu'à ne préparer que les grands jours et parfois par 'snobisme'. Le nkui (sauce gluante) correspond tout comme le taro (sauce jaune) aux Bamiléké. Dans le même sens l'évocation de la 'boule de mil' suffit pour déclencher une ascension vers les provinces septentrionales du pays. Et avec l'achu (autre sorte de taro) elles redescendent vers le Nord-Ouest; l'ékwan (macabo rapé) fait progresser vers le Sud-Ouest, alors que le ntuba (plantain pilé) les ramenera dans la province du Sud, etc. Ces tendances sont assez générales parce qu'à l'intérieur même d'une province (voire.carte à la page suivante), les groupes culturellement différents s'auto-identifient par d'autres aliments. C'est ce que nous allons essayer de montrer sur la côte sud-camerounaise.

\section{Cosmopolitisme de la côte et répartition spatiale}

La côte camerounaise dont il est question est celle située par $2^{\circ} 20$ à $3^{\circ} 20$ de latitude Nord et $9^{\circ} 50$ à 10 de longitude Est. Couverte par la grande forêt méridionale, cette région regorge de grands cours d'eau affluents de l'océan atlantique. Plusieurs groupes ethniques habitent la région côtière. Malgré les différences culturelles entre les uns et les autres, on peut procéder à un triple regroupement de ces entités cosmopolites sur la base de leurs activité et de leur mode d'occupation de l'espace (Visser, 1987). Ainsi aurons-nous:

- le groupe de chasseurs-cueilleurs ayant pour fief la forêt: il est constitué de Pygmées Ba-kola, environ un millier d'âmes. Les autres populations de la région les désignent par différents termes (Beyala chez les Mvae, Likoya chez les Yasa) et les colons ont vulgarisé les ethnonymes de Bagielli et Boyaelli (cf. Loung, 1987). Ces Pygmées vivent essentiellement de la chasse et de la cueillette. Ils s'organisent en groupements mobiles ayant pour vocation la chasse. Les membres d'un groupement sont issus de différents clans exogames qui constituent l'unité sociale des Pygmées (Dugast, 1949:6). La vie matérielle dans ces campements est assez rudimentaire et les conditions hygiéniques sont 'déplorables' (Ngima Mawoung, 1987:51). Contre leur gré, les Pygmées sont aujourd'hui contraints par les autorités administratives de mener une vie sédentaire. Dans ces nouvelles localités qu'on peut observer par endroit le long de la route Kribi-Campo, les Pygmées s'adonnent médiocrement à l'agriculture. Ils ne ratent jamais une occasion de retourner momentanément dans la forêt pendant certaines périodes de 


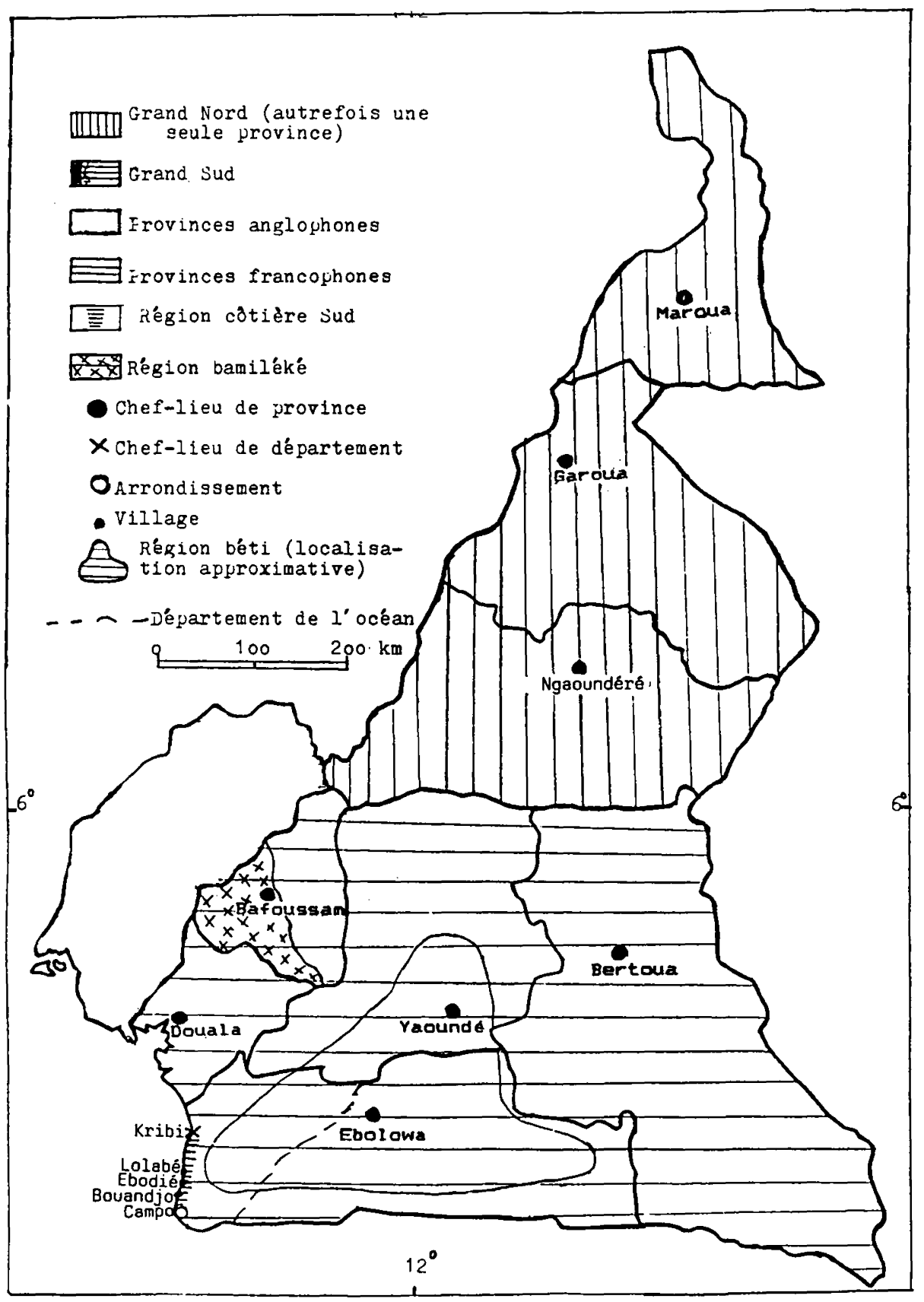

Carte 1: Localisation de quelques groupes de population 
chasse. Ils y restent 3 à 4 mois, avant de revenir languir au bord de la route, dans l'attente de la prochaine campagne de chasse.

- le groupe d'agriculteurs ayant aussi pour domaine la forêt: il s'agit des Mvae, des Mabéa et des Fang. Ce sont les peuples de la forêt qui vivent dans les villages les plus rapprochés de l'océan. Ils cohabitent dans certains cas avec les Yasa, mais occupent toujours le côté 'forêt' des villages. Environ une dizaine de mille, ils sont organisés en grandes unités exogames ou clans. Ils pratiquent accessoirement la chasse surtout en tendant des pièges, comme activité complémentaire à l'agriculture.

- le groupe de pêcheurs dont la spécialité est la mer: ce sont les Batanga et les Yasa. Ils vivent tout le long de la mer et ont pour activité principale la pêche. Les Batanga sont plus de cinq mille alors que les Yasa, groupe minoritaire sur la côte, sont estimés à un millier de personnes. Cette minorité ethnique est pratiquement inconnue sur la scène politique nationale. Traditionnellement, les Yasa sont une société acéphale, qui rejette l'institutionnalisation du leadership (Clastres, 1974); la légitimité politique est éphémère et on ne la reconnaît occasionnellement à un leader que tant qu'il se montre généreux. Les groupes sont constitués de lignages ayant chacun un ancêtre fondateur. La forme de mariage pratiquée est l'endogamie; ici on redoute la femme étrangère et entretient une sorte de mépris pour les non-ichtyophages parce qu'ils ignorent l'art de cuire le poisson.

\section{Les stratégies alimentaires dans la région}

Bien que ces différentes ethnies vivent dans le même environnement, leur mode d'appropriation de l'espace et leur choix des ressources alimentaires sont variés. La référence au monde naturel (espèces animales, nourriture, etc.) peut constituer, comme l'explique Claude Lévi-Strauss, un moyen d'ordonner l'univers social, de créer ou de penser au groupe, et d'articuler les relations entre les différents groupes (Lévi-Strauss, 1966). Les Pygmées qui ont pour fief la forêt ont basé leur stratégie alimentaire sur la viande. Ils l'accompagnent de tubercules qu'ils déterrent dans la forêt ou qu'ils obtiennent en échangeant la viande avec les ethnies voisines.

Les Mvae, eux, vont dans la forêt pour pratiquer l'agriculture. Ils cultivent et consomment différentes espèces végétales. Pour avoir le complément protéique, indispensable dans leur alimentation, ils font aussi un peu de chasse ou obtiennent 
le gibier ou le poisson respectivement auprès des Pygmées et des Yasa en leur livrant du manioc (Koppert \& Hladik, 1989:60).

Les Yasa, pêcheurs, sont ichtyophages par excellence. Autrement dit leur stratégie alimentaire est basée sur le poisson et d'autres produits de la mer. Les femmes produisent le manioc et en font des bâtons pour accompagner les sauces de poisson. Toutefois une bonne quantité de manioc est obtenue auprès des femmes mvae ou fang qui ont les meilleures capacités agricoles dans la région.

On peut donc constater que ces groupes ethniques voisins ne pratiquent pas d'autarcie alimentaire. Malgré la faible 'masse monétaire' dans cette région peu branchée à l'économie de marché (faible production des cultures de rente), les échanges alimentaires inter-ethniques sont assez poussés. Les différents groupes ont très souvent recours au troc pour faire circuler les trois produits stratégiques que sont la viande (Pygmées), le poisson (Yasa), le manioc (Mvae). Ce sont ces trois produits qui sous-tendent l'interdépendance entre eux. Et nous verrons qu'aucun de ces produits ne fait l'objet de mépris. Les stéréotypes ethnocentriques qui courent sur l'alimentation touchent plutôt les autres denrées.

\section{La symbolique du manger: nourriture et ethnicité yasa}

Dans leur répartition sexuelle des activités, l'homme yasa pêche alors que la femme cultive le manioc. Ce tubercule peut être préparée sous plusieurs formes: bâtons, tubercules ou couscous. Toutefois les Yasa ont une préférence marquée pour les bâtons de manioc. L'essentiel du temps de la femme yasa va dans la culture et la préparation du manioc sous forme de bâtons (cf. Pasquet, 1989). Deux fois par semaine, les femmes yasa rouissent le manioc à la rivière. Cette macération dure deux jours et a pour but l'élimination des principes toxiques amers (glucoside cyanogénétique), car 'l'imparfaite détoxication du manioc amer accentue la tendance au goitre dans les régions forestières sur socle cristallin où l'iode fait défaut' (Garine \& Bahuchet, 1989:51). Après cette opération les tubercules sont égouttés puis transportés à la maison où ils seront broyés et tamisés. Du produit obtenu, les Yasa modèlent des bâtons dans les feuilles de bananiers ou de marantacée. Ils les lient ensuite à l'aide d'une fibre de tronc de bananier. La seule confection des bâtons occupe la femme pendant toute une journée. 
Les plats sont accompagnés de sauce de poisson préparée avec du piment, du sel, et parfumée au jus de citron. C'est là un plat fort prisé chez les Yasa. Il est consommé tous les jours avec le même appétit.

Aucun autre aliment, quel que soit son degré de prestige ailleurs, ne peut facilement supplanter le plat traditionnel des Yasa. C'est par le poisson et le manioc que le Yasa se définit et tant qu'il n'en a pas mangé il n'éprouve pas grande satisfaction de sa satiété. Consommer d'autres aliments sont pour le Yasa une façon de tromper la faim. Ceux des Yasa qui ont un niveau de revenu élevé ont tendance à se procurer les aliments qui ne sont pas localement disponibles; cette pratique que I. de Garine désigne par 'snobisme alimentaire' vise à exhiber son statut économique. Mais en aucun cas il n'est question d'abandon de l'aliment culturel comme chez les Massa du Nord Cameroun, qui adoptent les comportements alimentaires, voire le style vestimentaire et la langue des Foulbés voisins, pour affirmer leur réussite (Garine, 1979:84). Même le ndolé (feuilles de vernonia amydalina), pratiquement devenu l'emblème de la cuisine nationale, n'est pas beaucoup apprécié des Yasa; ce rejet est dû au fait que les légumes sont en général chargés d'une valeur symbolique négative chez ces pêcheurs. Le plat de légume est synonyme de rareté ou d'absence de poisson. En effet la sauce de légume n'est pas 'découverte' tant qu'on n'y a pas plongé une cuillère pour la 'fouiller'. C'est pourquoi les Yasa voient dans le ndolé une façon de dissimuler le manque de poisson dans le plat. Ordinairement le plat yasa est 'découvert', parce qu'il contient peu de sauce et plus de poissons. Ce ndolé, pourtant connu des Yasa de longue date, est plutôt utilisé comme remède pour des soins gastriques et épidermiques: 'devant la pénurie croissante du poisson à l'ancienne Camerounville (aujourd'hui Campo) les habitants de cette ville cosmopolite ont estimé qu'ils pouvaient se servir de cette légumineuse pour leurs soupes, de telle sorte que le "ndolé" a changé de bord' (d'après les Lettres... de Djokou Mondjéli, non datée). Les Yasa ont une façon raffinée de préparer le manioc. Ils le broient et le tamisent avant de le modeler en bâtons, ce qui lui confère un goût nettement meilleur, par rapport à d'autres types de bâtons de manioc non tamisé avant leur modelage, qu'on retrouve à différents endroits du Cameroun. Le poisson est aussi cuit tout spécialement avec du citron, du sel et du piment. Cet art de cuire poisson et manioc dans lequel les Yasa sont passés maîtres fait d'ailleurs leur fierté auprès de leurs voisins. S'il faut parler comme Jelliffe (1967) de 'superfood', c'est-à-dire d'une nourriture sur laquelle une population est culturellement et économiquement concentrée comme étant la denrée principale (cf. McElroy \& Townsend, 1989:182), c'est que le poisson et le manioc en sont vraiment une chez les Yasa. C'est par cette nourriture que les Yasa se distinguent des autres ethnies et répondent à leurs stéréotypes ethnocentriques: 'Pour les Yasa du sud Cameroun leurs voisins, les Mvae, sont 'des paysans de l'intérieur, mangeurs de feuilles qui 


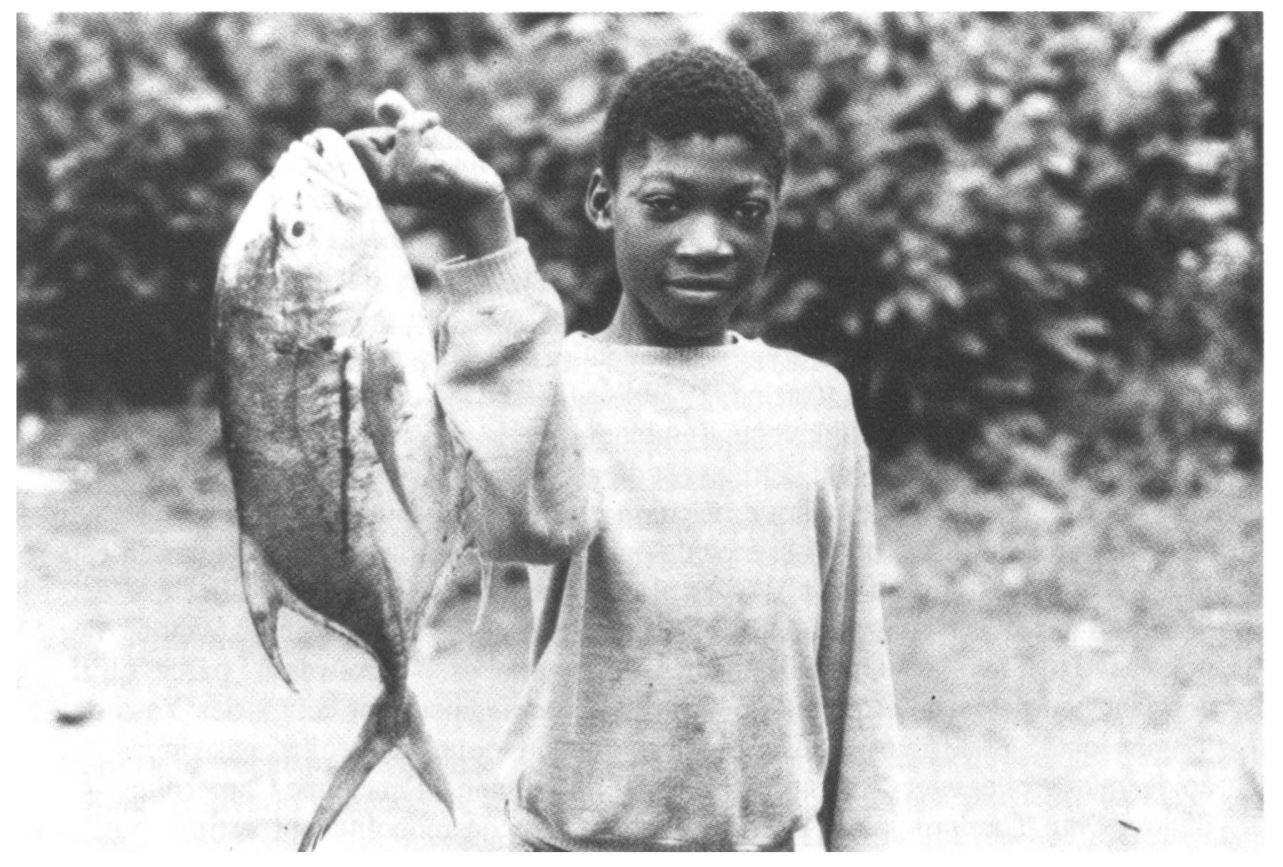

Photo 1: Un jeune Yasa avec sa capture. 
viennent quémander le poisson'. Pour les Mvae, les Yasa apparaissent comme de 'piètres agriculteurs, mangeurs de fruits de l'arbre à pain et de crabes dont eux mêmes méprisent la consommation' (Garine, 1989:80).

\section{La guerre des stéréotypes alimentaires}

Quand les membres des différents groupes sont en présence des uns les autres ils utilisent rarement les stéréotypes pour se qualifier. Ils se respectent mutuellement. C'est surtout lorsqu'on est dans des groupes homogènes qu'on entend le plus souvent des stéréotypes sur d'autres. Nous avons signalé que ces clichés ne touchent pas aux produits stratégiques (viande, poisson, manioc) qui constituent la base des échanges entre ces ethnies. Les denrées qui sont concernées sont celles dotées de peu de prestige ou celles répugnées par chaque groupe, et pour lesquelles on voudrait déclencher un 'dégoût universel' (cf.Douglas, 1979:145).

- Les fruits de l'arbre à pain. Pour les Mvae, bons agriculteurs, ces fruits qu'on cueille sont le symbole de la paresse. Ils se moqueront des Yasa en disant qu'ils sont les 'mangeurs' de ces fruits. Les Yasa, qui ne veulent pas être un objet de risée, cueillent les fruits de l'arbre à pain mais les gardent pour des échanges avec les Pygmées. Ils sont d'excellents pêcheurs et ne cachent pas leur manque de motivation pour les activités agricoles, qu'ils justifient par l'hostilité du milieu: la forêt dense non remaniée par l'intervention de l'homme (cf. Tiokou Ndonko, 1988:8). Néanmoins les femmes yasa pratiquent une agriculture de subsistance (monoculture de manioc) sur de petites unités de moins de 0,5 ha.; ce que n'arrivent pas à réaliser les Pygmées. Pour cette raison les Yasa leur réservent les fruits de l'arbre à pain qu'ils considèrent, à leur tour, comme signe de la nullité des capacités agricoles. Les Pygmées ne demandent pas mieux et supportent -à contrecoeur- le déséquilibre de la balance du troc pour obtenir de quoi compléter leur ration de viande.

- Les crustacés. Les Mvae mangent aussi du poisson mais répugnent à la consommation des autres crustacés, notamment les crabes que consomment les Yasa. Pour affirmer ou manifester la différence d'avec les Yasa, les Mvae les cataloguent comme les 'mangeurs de crabe'. Et comme les Mvae redoutent l'environnement maritime et doivent passer par les Yasa, pêcheurs, pour obtenir du poisson, ces derniers les traitent alors de 'quémandeurs de poisson'. Notons aussi que les aliments qu'on repousse sont parfois l'objet de prohibition. Mentionnons à ce sujet l'exemple des Bafia (Centre du pays); dans ce groupe il 
existe un tabou très fort touchant la tortue, qui symbolise le mal: non seulement il est interdit de consommer cet animal mais on ne doit ni le voir ni en parler; toute transgression déclenche un type de maladies diarrhéiques très grave et redouté par tous les Bafia. Cette maladie, qui peut toucher la descendance même après plusieurs générations, est connue sous le nom de kebène ke kechek ke kui, c'est-à-dire la 'diarrhée due au sort causé par la tortue' (Tiokou Ndonko, 1989: 2-3).

- Les feuilles (légumes). Les Yasa sont ichtyophages et les légumes sont pour eux le signe d'une 'crise' de poisson. Les Mvae, eux, cultivent différentes espèces végétales et consomment plusieurs types de feuilles ou légumes. Les Yasa les traitent à cet effet de 'mangeurs de feuilles'.

Voilà donc des groupes qui vivent côte à côte, mais qui ont tellement 'culturalisé' la nourriture qu'ils finissent par se distinguer comme des 'mangeurs' de ceci ou de cela. Comment ne pas croire dès lors à l'affirmation de Luce Giard, d'après laquelle 'Rien n'est plus variable d'un groupe humain à l'autre que cette notion du mangeable' (Giard \& Mayol, 1980:173-4; V.aussi Harris, 1987). C'est dans le même sens que nous avons au début de ce texte cité un extrait de Hilton-Simpson sur la cynophagie et l'anthropophagie. En dehors de ces stéréotypes qui courent sur l'alimentation il existe aussi dans chaque groupe un lexème qui permet de s'auto-différencier des autres ethnies. Selon le contexte, ce terme peut revêtir une connotation péjorative ou méliorative. Chez les Yasa, par exemple, on parle de pogn pour désigner tous ceux qui sont non-Yasa, c'est-à-dire les autres peuples de l'intérieur. Les Yasa diront par exemple d'un Mvae qui défèque dans la brousse que c'est un pogn; ce qui permet de le mépriser et surtout de l'excuser. L'Homme yasa, lui, ira plutôt au bord de la mer pour ce besoin; cet endroit étant la zone de défécation socialement admise et recommandée. Les Yasa croient que là on apporte sa modeste contribution dans la chaîne alimentaire en nourrissant les poissons de son riche excrément (cf. Tiokou Ndonko, 1988b); même l'acte apparemment banal de déféquer permet ainsi aux individus de manifester leur appartenance au groupe yasa. Le pogn n'est donc pas seulement un 'quémandeur' de poisson, c'est aussi celui qui gaspille la 'nourriture' des crustacés dans la brousse et les latrines. Le terme pogn qui appelle ainsi au mépris et à la tolérance des comportements déviants par rapport aux normes de la société yasa, est aussi utilisé à l'intérieur même du groupe yasa pour ridiculiser quelqu'un. C'est ainsi qu'un Yasa appellera un autre pogn s'il boutonne mal son habit par exemple, et ceci, pour lui faire comprendre que ça ne se fait pas ainsi. Il y a dans cette attitude un rappel à l'observance des normes et une tendance à ériger un modèle de comportement à l'échelle collective. On peut se référer à Michel Foucault (1969) pour mieux 
appréhender cette façon de procéder, et même la manière de penser des institutions (Douglas, 1987).

\section{Du quartier à la Nation: le Yasa hors du village}

\section{Identification}

Nous voulons dans ce qui suit discuter l'identité ethnique dans un tout autre contexte, celui de la présentation de soi. Lorsque le Yasa est hors du village ou de la région, il manifeste autrement son ethnicité. A l'inévitable question, qui accompagne les salutations au Cameroun: 'tu es quoi?', 'tu viens d'où?', le Yasa, s'il est à Campo -principale localité de l'arrondissement- répondra ainsi: je suis Yasa d'Ebodié ou de Bouandjo ou de Lolabé, etc. A ce niveau les villages sont plus ou moins connus et c'est pour cela que l'ethnicité est localisée dans le cadre spatial du village. Si l'interlocuteur est aussi Yasa, l'auto-identification se fera par déduction de l'ethnicité au lignage, en passant par le village.

De cette façon toutes les structures administratives sont récupérées et servent de repérages spatiaux dans le processus d'induction-déduction d'auto-définition de soi. Au niveau du département (Kribi) le Yasa se définit dans cet ordre:

$$
\begin{aligned}
& \text { - je suis de Campo } \\
& \text { - ... Yasa } \\
& \text { - ... d'Ebodié / de Bouandjo (par exemple). }
\end{aligned}
$$

Il ne progresse dans la déduction ou la singularité que tant que son interlocuteur se retrouve dans le système de repérage spatial. Au niveau de la province (Ebolowa), le Yasa remontera dans l'induction et parlera en premier lieu du département d'origine, l'Océan. Dans une province anglophone ou une province septentrionale du pays, la première identité varie: le Yasa se définira ou sera identifié d'abord comme francophone, ou comme sudiste. Il faut peut être noter le fait qu'à l'extérieur et surtout dans les milieux diplomatiques, on aime voir fonctionner le Cameroun sous le modèle Nord-musulman, Sud-chrétien. Il ne faut pas se méprendre car il existe autant de fidèles du christianisme et de religions 'traditionnelles' dans le Nord qu'il existe de musulmans et autres animistes dans le Sud. 
Ailleurs sur le plan national, le Yasa pour se présenter commencera par:

- ... Province du Sud

- ... Côtier / Kribien.

Ce n'est que si on l'assimile à un Batanga que le Yasa à ce niveau précisera son groupe ethnique. Signalons que pour beaucoup de Camerounais des provinces autres que le Sud, 'Kribien' est synonyme de Batanga. Cette généralisation abusive est due au fait que la ville de Kribi -chef-lieu du département- est le fief des Batanga et aussi parce que ces derniers sont le groupe dominant sur la côte sud et donc le plus connu. Quand le Yasa dit qu'il est 'Kribien' il y a aussi derrière cette identité un besoin de ne pas révéler son ethnie véritable; il ne donne d'amples précisions que parce qu'il se sent acculé. On retrouve ce genre de 'dissimulation' dans la province du Littoral où les originaires de différents groupes ethniques disent carrément qu'ils sont Duala. Ce qui pousse les individus à se réclamer d'autres groupes ethniques, c'est parfois une image peu flatteuse qui est véhiculée au sujet de leur village ou région d'origine. Les Camerounais ont parfois honte de leur village de brousse situé à la périphérie de la table du développement où se mange le 'gâteau national'.

Hors du territoire national le Yasa répond tout simplement qu'il est Camerounais. Il ne précise un peu plus son identité que s'il a affaire à un compatriote ou à un interlocuteur qui a une idée du Cameroun. Je voudrais, pour étayer ce fait, rappeler la substance de ma première entrevue avec un Yasa à Sarh, dans le Sud du Tchad au début d'Avril 1990:

- Ah, vous êtes Camerounais! Moi aussi. Vous êtes d'où?

- Bamiléké

- Eh! Il y a des compatriotes ici, un couple bamiléké; ce sont de très bons amis. (...) Moi, je suis Côtier de Kribi.

- Tiens! Je connais bien cette région.

- Ah bon! Tu connais l'Océan? (le département de)

- Oui (...) J'ai séjourné à Ebodié.

- Ce n'est pas vrai? Tu connais Ebodié? Ce n'est pas possible!

- J'y mène des recherches depuis trois ans.

- Mais, c'est mon village. Je suis Yasa d'Ebodié.

- C'est formidable!

- Dis, as-tu mangé les bâtons et le poisson? Chez qui habites-tu au village?...

Cette petite conversation illustre bien le mécanisme d'induction-déduction qui rentre en jeu dans l'auto-identification et révèle clairement que l'ethnicité n'est 
qu'une phase de ce processus. On se sert parfois du repère anthroponymique au Cameroun pour classer les individus et se situer dans l'une des phases du processus d'identification (région, ethnie, province, département, arrondissement, village, quartier, voire clan ou lignage). Cette démarche consiste à partir d'un nom propre pour remonter l'ethnicité d'un individu, voire aller plus loin en précisant son village, son quartier ou son clan (cf. Voorhoeve, 1964; Delatour, 1975; Tiokou Ndonko, 1986). L'objectif est donc toujours de situer l'individu dans une 'classe préordonnée' (Lévi-strauss, 1962:240).

\section{Urbanité, ethnicité et classes sociales}

Dès l'aube des indépendances les romanciers camerounais, qui devaient plus tard être relayés par des sociologues, économistes et démographes, ont relevé pour s'en indigner l'orientation structurelle du nouvel espace urbain. C'est que la ville était un lieu de confrontation des intérêts socio-politico-économiques entre le 'colon blanc' et l' 'indigène noir'. On ne peut pas comprendre le phénomène urbain dans le Cameroun d'aujourd'hui en occultant cette ambivalence historique.

Le colon, plus ou moins parti, a cédé sa place et l'espace aux nouveaux 'ducs' du pouvoir néocolonial. Ils occupent les quartiers tracés, richement construits et au paysage verdoyant. Là se trouvent non seulement les privilégiés des régimes qui se succèdent mais aussi leurs différents conseillers et experts internationaux. De l'autre côté on a la masse des 'citadins-ruraux' entassés dans les quartiers populeux et insalubres. Entre les deux se trouvent la petite bourgeoisie des fonctionnaires moyens qui occupent des 'camps' ou 'cités' aménagés.

Dans les quartiers pauvres les ressortissants de la 'brousse' renouent avec certaines valeurs de leur terroir telle que l'entraide. Ils sont largement influencés par leur 'ethnicité' dans l'exercice d'une activité ou leur installation dans tel ou tel sous-quartier. Même si on assiste de plus en plus à un brassage entre les ethnies (Ela, 1983:56) le principe de différenciation des groupes demeure l'ethnicité. Ils le remontent, comme nous l'avons déjà montré, dans le sens du village, voire du quartier ou du lignage selon que l'interlocuteur se retrouve dans le processus. Dans ces milieux les plats traditionnels sont encore largement consommés, même si des denrées nouvelles comme le pain, le riz, etc. qu'imposent la vie urbaine sont incorporés à l'alimentation. Dans les quartiers riches en revanche il y a une tendance, pas à la renonciation, mais au dépassement de l'ethnicité. De nouvelles valeurs tel l'argent, le pouvoir apparaissent et permettent aux individus de se retrouver dans des associations non-ethniques; ce qui n'exclut pas pour autant leur 


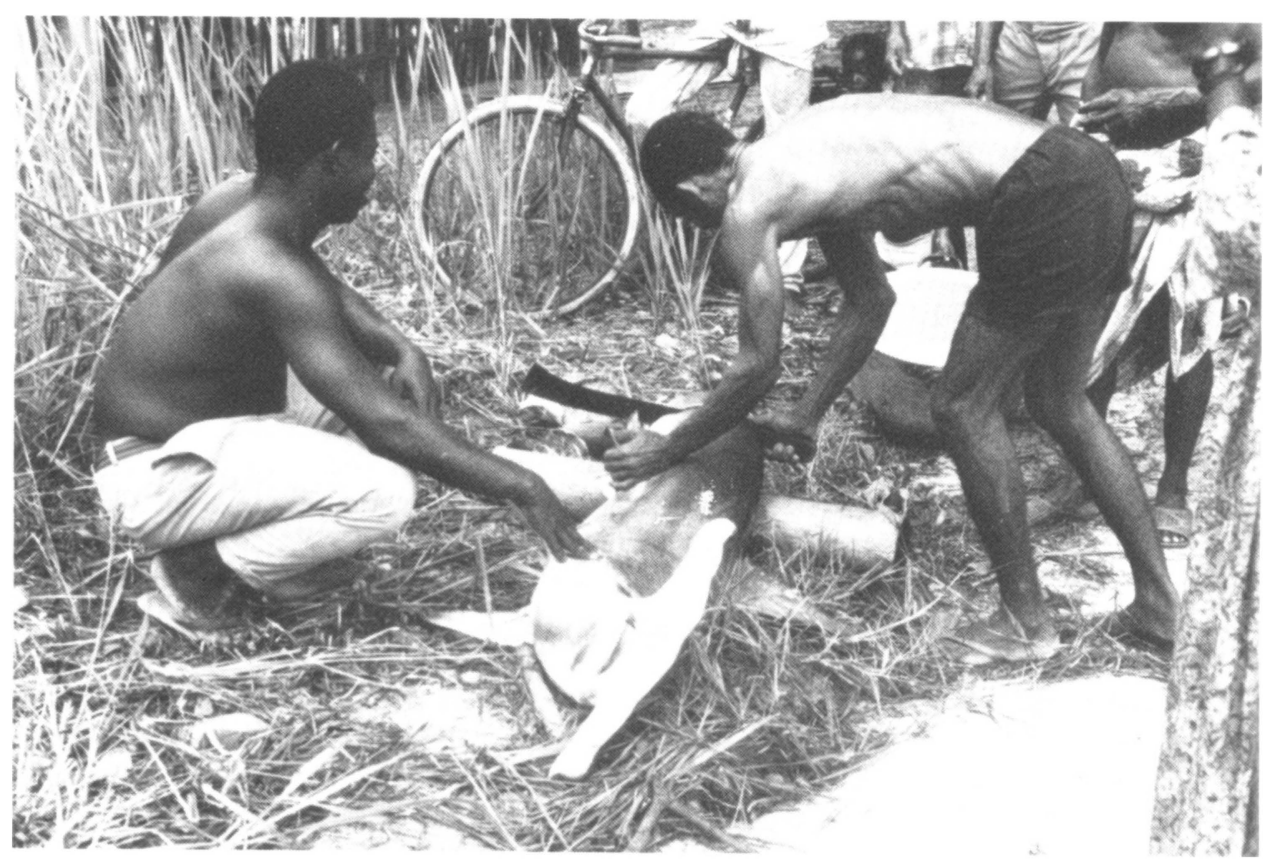

Photo 2: Des pêcheurs découpent un marteau (requin) au bord de la mer. 
participation à d'autres formes de regroupement à base ethnique. L'argent permet ici non seulement d'affirmer son statut socio-économique mais aussi de manipuler des ressortissants du village à des fins socio-politiques; ces manipulations se font assez souvent par la distribution de la nourriture ou de la boisson, et de plus en plus aussi par l'argent. Les élites utilisent leur identité ethnique d'une manière très intéressée. Il n'en est pas de même chez leurs descendants où l'ethnicité tend à devenir amorphe. Les fréquentations chez ces derniers se font en général en dehors des considérations ethniques; les enfants vont voir les fils des collègues ou des amis à Papa et Maman. Leur amitié est parfois motivée ou se manifeste par le désir de jouer au tennis, de fréquenter les 'boîtes' (de nuit) luxueuses ou de conduire la 'Merco' (Mercedes) de Papa. A la traditionnelle question: 'Tu viens (es) d'où?' se substitue la suivante: 'Que fait ton Pater /ta Mater?'. Le fils d'un Médecin (Ewondo) trouvera plutôt un intérêt à fréquenter celui d'un Professeur (Bamiléké) ou d'un Ingénieur (Babouté) que celui d'un Chauffeur (Ewondo), même si ce dernier conduit le Ministre. La tendance à s'associer ici est fondée sur des affinités de classe. On peut observer, à la sortie des classes, dans les grands établissements scolaires des villes que certains 'enfants de Boss' -comme les appellent les moins nantis- transportent leurs petit(e)s ami(e)s du quartier et non les 'sous-développés' qui vivent dans les quartiers sans route.

Plus le statut social des individus est élevé et varié, moins on se sert de l'ethnicité pour les classer. On parle volontiers de fils du Ministre, du D.G.(Directeur Général), du Colonel, du Commissaire etc. pour différencier ou identifier les individus. Dans ces milieux on raffole du 'camembert' ou 'caviar' et non du 'tapioca'. On n'utilise plus la langue, considérée comme dégradante, du terroir. Les valeurs qui expriment l'ethnicité sont peu agissantes et le snobisme touche ici non seulement à l'alimentation mais aussi aux façons de parler, de se loger, se vêtir, se divertir, etc. L'identité ethnique est plutôt récupérée à des fins purement utilitaires (Cf. Bayart, 1985, 1989).

\section{De l'usage politique de l'ethnicité: pluralisme politique et mobilisation ethnique}

Le principe d'ethnicité n'est pas par essence un principe déflagrant; il n'explose que lorsqu'on y met du feu. On ne peut pas le gommer -même pas par décret, fût-il d'un Président- car il correspond à un besoin institutionnel, le besoin de rassembler, classer et identifier les individus. De nombreux gouvernements africains ont, dès le lendemain des indépendances, perçu en les différences ethniques un danger pour l'édification de leur Etat unitaire. Ils n'ont ménagé aucun 
effort pendant plus d'un quart de siècle pour chasser les 'démons du tribalisme', les 'champions de la division'. On ne peut que s'étonner que presque 30 ans après il soit encore question de 'démons'.

C'est qu'en réalité l'ethnicité est devenue pour de nombreux pouvoirs postcoloniaux une stratégie politique. On joue la carte ethnique soit pour mobiliser, soit pour démobiliser en sa faveur. L'observateur averti de la scène politique camerounaise ne manquera pas d'établir une corrélation entre le pluralisme politique et la mobilisation ethnique. Ce qu'on appelle ici 'tribalisme' n'est jamais aussi fort que lorsqu'il y a différence d'opinion sur les grandes questions politiques. Notons d'emblée que les différents ethnonymes ne sont pas toujours la création des Camerounais eux-mêmes. Les premiers colons, eux aussi, dans le besoin de répertorier les populations, leur ont plaqué des étiquettes diverses selon leur habilité à entendre et transcrire les positions géographiques, les noms de personnes, etc. dans les langues de leurs guides (cf.Dongmo, 1981:10,45; Loung, 1987). Puis on a cultivé l'appartenance aux différents ensembles en apprenant aux populations leur 'race'. Même après les indépendances, on continuera à porter sa 'race' - à entendre comme ethnie - sur les papiers officiels au Cameroun. Et quand commence la construction de l'unité nationale la race est gommée, les partis politiques aussi. Le moins qu'on puisse dire des chercheurs est qu'ils n'échappent pas à ce genre de manipulation ('identificatoire'). Nous pouvons citer trois petits exemples pour ce qui est de la Côte: les deux premiers sont Ardener (1956:10) et Bureau (1962:17-8) qui regroupent comme ils peuvent les 'Côtiers'; (pour ne pas parler que des autres) le troisième exemple c'est bien le nôtre: n'avons-nous pas procédé au début de cet article à un 'triple regroupement' des groupes ethniques dont nous parlons?

La première grande mobilisation de la 'carte ethnique' se situe à la veille des indépendances. Pour contrecarrer l'implantation des partis nationalistes les autorités coloniales ont en partie recouru à l'argument ethnique. L'Union des Populations du Cameroun (U.P.C) qui les inquiétait à l'époque était présentée comme le parti des Bassa et des Bamiléké (cf. Joseph, 1986; Bayart, 1985). Par ce moyen elles ont procédé à une démobilisation générale dans les parties du pays où l'U.P.C. n'était pas encore enracinée et à la création des 'contre-partis' à base ethnique. C'est dans ce contexte que démarre 'l'unité nationale' qui a alors comme pierre angulaire le parti unique.

Aujourd'hui on assiste à une résurgence démocratique au Cameroun. Le parti unique d'hier s'est préparé pour la 'concurrence' et a autorisé le fonctionnement légal d'autres sensibilités politiques. Du côté de l'opposition comme de celui du pouvoir, on pointe les visées 'tribalistes'. D'un côté le pouvoir est accusé 
d'ethniciser les postes-clés, de l'autre l'opposition est accusée de vouloir secouer la carte tribale. Il a même récemment été question de 'milice ethnique'. Ainsi le politique récupère le besoin d'ordonner inhérent aux institutions et aux peuples pour semer la haine, transformer les 'mangeurs de...' en 'tueurs de...'.

Nous ne saurons conclure notre propos sans évoquer quelques conflits à caractère plus ou moins ethniques -sujet tabou au Cameroun, qui est étonnamment l'un des pays les plus stables du monde, compte tenu de l'ampleur des différences qui existent entre ses populations- qui ont déjà fait quelques victimes. Ce sont les affrontements Foulbé-'Kirdi' (label collé aux populations non-musulmanes des montagnes du Nord du pays), Foulbé-Gbaya qu'on explique vulgairement comme motivés par les différences de religion; il y a aussi le schéma oppositionnel Sudiste-Nordiste, Anglophone('Anglo')-Francophone('Frog'); dans les parenthèses c'est la terminologie des campus universitaires où les 'affrontements' éthnocentriques verbaux sont le lot du quotidien. On a également des oppositions Tikar - Mbororo, Pygmées - 'Autochtones' ou 'Villageois' et de mieux en mieux Bamiléké ('Bami' ou 'Glafis') - Béti ('Nkoua'). Nous nous inscrivons en faux contre la réduction de ces contrastes à une simple haine. On ne peut sérieusement les étudier qu'en reconsidérant le contexte global de la société avec ses enjeux politico-économiques, religieux et fonciers. Pourquoi ces conflits ne seraient pas aussi une réaction tactiquement détournée du peuple contre un Etat qui ne sait pas toujours se passer de l'arme de la répression, en vue d'exprimer son insatisfaction. On l'a vu avec les 'ripostes paysannes à la crise' (Ela, 1990).

\section{Conclusion}

En conclusion, l'ethnicité correspond à un besoin de regrouper, de classer ou d'ordonner les populations. Ce besoin est presque naturel aux institutions ou aux membres d'une communauté. Les groupes se donnent ou cherchent à se donner une identité pour mieux contrôler leurs membres et les sanctionner si ce besoin se fait sentir. L'ethnicité permet aussi face aux 'autres' de régler la flexibilité des normes afin de garantir leur protection et permettre ipso facto la tolérance. C'est par elle que s'effectue l'organisation et la reconnaissance du droit de tous et de chacun à l'espace. A travers le poisson et le manioc, nous avons vu comment un groupe minoritaire sur la côte camerounaise, les Yasa, gère la différence et affirme sa spécificité. Malgré la 'guerre' des stéréotypes alimentaires et des préjugés ethnocentriques avec les groupes ethniques voisins, ils vivent tous assez paisiblement les uns et les autres. Tant que le politique ne s'y est pas mêlée pour cultiver 
la haine en vue d'enjeux divers, ces ethnies de l'arrière pays, qui ont en commun la pauvreté, n'auront toujours pour principe de différenciation que les façons de gérer l'espace et de manger qui les distinguent.

\section{Bibliographie}

ARDENER, E. (1956). The coastal Bantu, London, International African Institute.

BAYART, F. (1985). L'Etat au Cameroun, Paris, Presses de la Fondation Nationale des Sciences politiques (2e éd.).

BAYART, F. (1989). L'Etat en Afrique: La politique du ventre, Paris, Fayard.

BUREAU, R. (1962). 'Ethno-sociologie religieuse des Duala et apparentés' in: Recherches et Etudes Camerounaises, no. 7-8: 17-372.

CLASTRES, P. (1974). La société contre l'Etat, Paris, Edition de Minuit-coll. Critique.

DELATOUR DEJEAN, C.-H. (1975). Parenté et famille dans une chefferie du Ndé (Cameroun), Thèse de Doctorat, Université de Paris $\mathrm{X}$.

DONGMO, J.L. (1981). Le dynamisme bamiléké (Cameroun): La maîtrise de l'espace agraire, Yaoundé, CEPER.

DOUGLAS, M. (1979). 'Les structures du culinaires' in: Communications, no. 31: 145-70.

DOUGLAS, M. (1987). How institutions think, London, Routledge \& Kegan Paul.

DUGAST, I. (1949). Inventaire ethnique du Sud Cameroun, Mémoires de l'Institut français d'Afrique noire, Série Populations, No. I.

ELA, J.-M. (1983). La ville en Afrique noire, Paris, Karthala.

ELA, J.-M. (1990). Quand l'Etat pénètre en brousse..., Paris, Karthala.

FOUCAULT, M. (1969). L'archéologie du savoir, Paris, Gallimard-N.R.F.

FRANK, B. (1965). Die Rolle des Hundes in Afrikanishen Kulturen, Wiesbaden, Franz Steiner Verlag GMBH.

GARINE, I. (1979). 'Culture et nutrition' in Communications, no. 31: 70-90.

GARINE, I. (1989). 'Organisation des repas, valeur attribuée aux aliments et structures socio-économiques' in: Se nourrir en forêt équatoriale, Paris, Unesco/MAB: 78-82.

GARINE, I. \& BAHUCHET, S. (1989). 'Conservation et préparation des aliments' in: Se nourrir en forêt équatoriale, Paris, Unesco/MAB: 50-2.

GIARD, L. \& MAYOL, P. (1980). L'invention du quotidien: Habiter et cuisiner, tome 2, Paris, Union Générale d'Editions 10/18.

HARRIS, M. (1987). The sacred cow and the abominable pig, (Riddles of food and culture), New York, Simon \& Schuster Inc.

JELLIFFE, D.B. (1967). 'Parallel food classifications in developping and industrialized countries' in: American Journal of Nutrition, no. 20: 279-81

JOSEPH, R. (1986). Le mouvement nationaliste au Cameroun, Paris, Editions Karthala (version française).

KOPPERT, G. \& HLADIK, C.M. (1989). 'Mesure de consommation alimentaire' in: Se nourrir en forêt équatoriale, Paris, Unesco/MAB: 59-62. 
LEVI-STRAUSS, C. (1962). La pensée sauvage, Paris, Librairie Plon.

LEVI-STRAUSS, C. (1966). Anthropologie structurale, Paris, Librairie Plon.

LOUNG, J.F. (1987). 'Le nom authentique du groupe Pygmée de la région côtière camerounaise' in Revue de Géographie du Cameroun, vol. 7, no. 2: 81-94.

McELROY, A. \& TOWNSEND, P.K. (1989). Medical Anthropology in Ecological Perspective, Boulder, Westview Press (2e éd.).

PASQUET, P. (1989). 'Bilan énergétique et adaptabilité nutritionnelle' in: Se nourrir en forêt équatoriale, Paris, Unesco/MAB: 64-66.

TIOKOU NDONKO, F. (1986). Anthroponymie chez les Bamena (Ouest Cameroun), Université de Yaoundé, Term paper (manusc.).

TIOKOU NDONKO, F. (1988a). Notes sur les Yasa, n.d.

TIOKOU NDONKO, F. (1988b). Représentations et pratiques culturelles liées aux excréments et autres déchets du corps chez les populations de la savane et de la forêt (côtière), Dissertation doctorale (D.E.A. en Anthropologie), Université de Yaoundé.

TIOKOU NDONKO, F. (1989). Rapport de participation à l'étude L.M.D. Cameroun, Pritech-Usaid (Arlington, Virgina 22209), Assign. no. DC 465.

VISSER, L. E. (1977). 'L'igname, bonne à manger et à penser: Quelques aspects de l'agriculture ahouan (Côte d'Ivoire)' in: Cahiers d'Etudes Africaines, 68, 17-4: 525-544.

VOORHOEVE, J. (1964). 'Notes sur les noms d'éloge bamiléké' in: Cahiers d'Etudes Africaines, no. 15 , vol. $3-4,: 452-455$. 


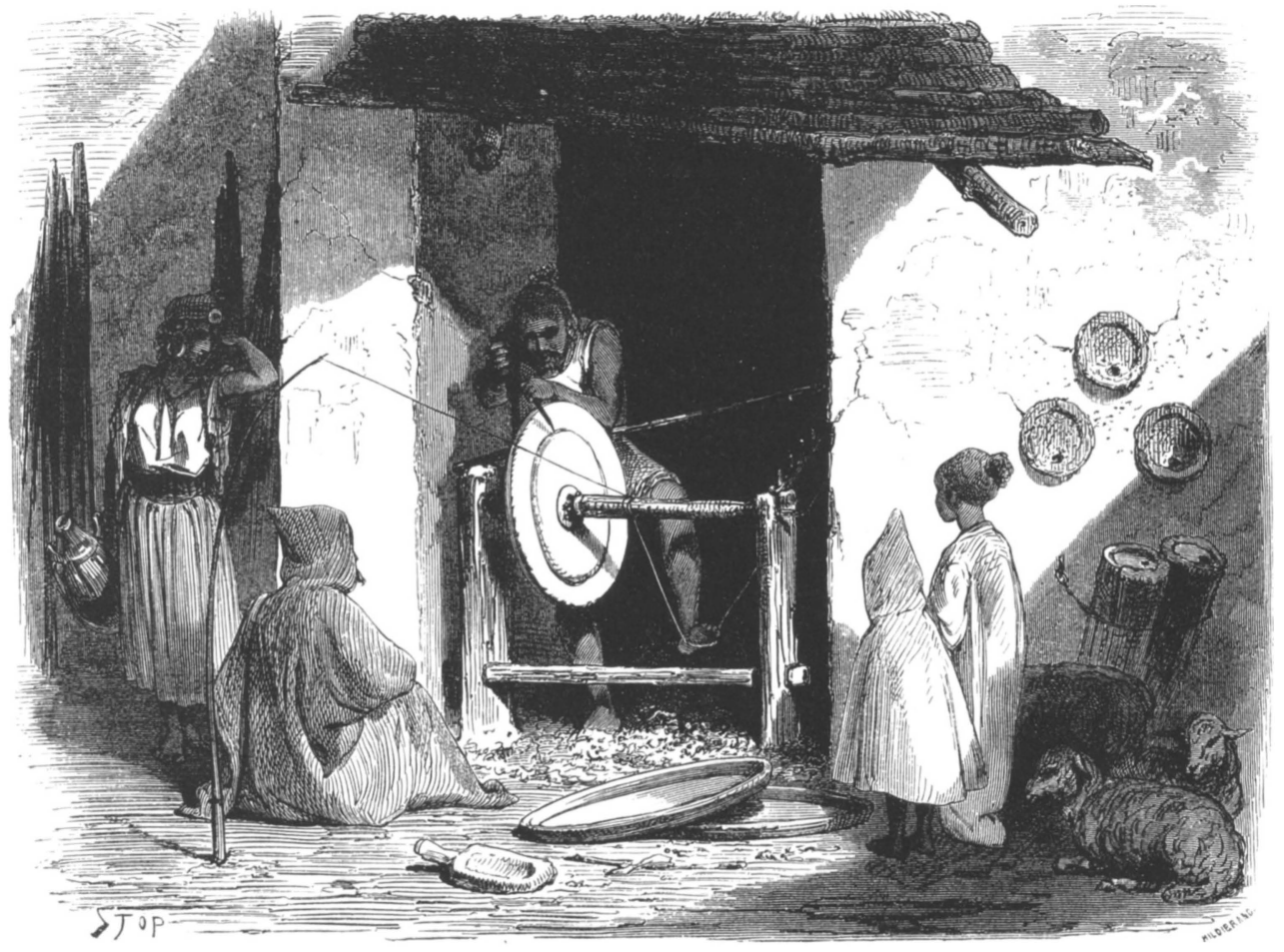

Tourneur kabyle. - Dessin de Stop d'après un croquis du commandant Duhousset.

\section{Kabylische houtdraaier}

Gravure uit: Commandant DUHOUSSET, Excursion dans la Grande Kabylie, notes et croquis receuillis entre la Méditerranée et le Djurjura. In: Le Tour du Monde. Nouveau Journal des Voyages, 1867 deuxième semestre, Paris, Hachette, p. 304. 